\title{
Serum leptin concentration in patients with type 2 diabetes
}

\author{
Arleta Malecha-Jedraszek ${ }^{1 *}$, Agata Burska ${ }^{3}$, \\ Beata Matyjaszek-Matuszek ${ }^{2}$, Helena Donica ${ }^{1}$
}

${ }^{1}$ Department of Biochemistry Diagnostics, Medical University of Lublin, Staszica 11, 20-081 Lublin, Poland
${ }^{2}$ Department of Endocrinology, Medical University of Lublin, Jaczewskiego 8, 20-090 Lublin, Poland
${ }^{3}$ Leeds Institute of Rheumatic and Musculoskeletal Medicine, University of Leeds, Beckett, LS9 7TF Leeds, UK

\section{ARTICLE INFO}

Received 20 October 2015

Accepted 23 October 2015

\section{Keywords:}

leptin, adipocytokines,

adipose tissue,

type 2 diabetes.

\begin{abstract}
With the increasing importance of early type 2 diabetes (DM2) and obesity detection, it is useful to reevaluate leptin role in these conditions. Our study aimed at investigating circulating leptin concentrations in a group of patients with DM2, and at assessing in detail whether leptin concentrations correlate with selected biochemical, clinical parameters and markers of systemic inflammation in patients with DM2 and in healthy volunteers. In our work, we analysed samples and data drawn from 71 patients aged $61.4 \pm 11.7$ years, who have been diagnosed with type 2 diabetes, as well as from a healthy control group (HC) consisting of 51 healthy subjects with a mean age of $57.8 \pm 13.7$ years. Therein, the concentration of leptin in the DM2 patients was significantly higher than in the HC $(\mathrm{p}<0.01$ ), with median value of 16.59 (IQR 8.58-33.39) $\mathrm{ng} / \mathrm{ml}$ in the DM2, vs median value of 6.66 (IQR 4.52-21.40) ng/ml in the HC. In the analysis of variance, higher leptin concentrations were revealed in the DM2 group as compared to the HC, and this figure remained significant after adjusting for gender and age $(\mathrm{p}<0.001)$. Moreover, it was independent of HOMA-IR $(\mathrm{p}=0.003)$. However, the differences in leptin levels between the groups disappeared when additional adjustments for anthropometric parameters (BMI, waist circumference) were applied $(\mathrm{p}=0.088)$. Beyond the aforementioned, significant positive correlations were found in the DM 2 group between leptin level and CRP $(r=0.256 ; \mathrm{p}<0.05)$ and IL-6 $(\mathrm{r}=0.345 ; \mathrm{p}<0.01)$. Among the selected variables, only gender and BMI were included in the predictive model explaining the variability of leptin, and, in total, were responsible for $72.6 \%$ of the original variation of the studied adipocytokine. The results of this study have led to conclusion that leptin may participate in the complex pathogenesis of DM2 and be a predictor of the development of this disease. As higher concentrations of leptin coexist with obesity, and this situation correlates positively with markers of inflammation (CRP, IL-6), leptin level, hence, should be considered in the pathogenesis of DM2.
\end{abstract}

\section{INTRODUCTION}

The diabetogenic impact of obesity on type 2 diabetes mellitus (DM2) has not been fully elucidated so far. Still, as obesity results from an imbalance between food intake and energy expenditure, which leads to an excessive accumulation of adipose tissue, a number of research studies on obesity have aimed at understanding the exact molecular mechanisms that lead to the development of "diabesity"

\footnotetext{
* Corresponding author

e-mail: a.malecha@wp.pl

tel.: +48 (81) 5323816
}

(obesity-dependent diabetes mellitus). There is an increasing interest in the role played by adipose tissue in the body as it is now recognized as being not only a passive reservoir of energy excess derived from food intake, but also as an active endocrine organ capable of synthesizing and releasing a variety of molecules. These adipose tissue derived bioactive molecules are known as adipocytokines or adipokines. Although the precise mechanisms are still unclear, dysregulated production or secretion of these adipokines induced by excess of adipose tissue and its dysfunction, can contribute to the development of obesity-related metabolic diseases. Multiple lines evidence provides valuable 
insights into the roles of adipokines in the development of obesity and its link to metabolic complications such as insulin resistance, DM2, dyslipidemia, hypertension, and atherosclerotic cardiovascular disease [2,3,13,17].

The first discovered adipocytokine is leptin. The name of "leptin" comes from the Greek word "leptos", meaning "thin", and it regulates the energy balance and food intake. This leptin function is also called "Adipostat", and originates in the hypothalamus (the central nervous system). It sets a defined pre-set weight for any individual. Leptin decreases orexigenic and increases anorexigenic peptide synthesis in the hypothalamus: thereby decreasing appetite/promoting satiety and increasing energy expenditure. Extensive knowledge about the importance of leptin and its role in the pathogenesis of metabolic disorders is still insufficient, because leptin remains a very important link between the amount of stored body fat and the multiple processes that are undergoing in the human body [7,22].

It has been postulated that, in addition to its neuroendocrine role in weight control, leptin also can modify the insulin sensitivity of various tissues, as well as $\beta$-cell function in the pancreas. Moreover, it may play a role in blood pressure control, as well as in immune system function, and take part in the inflammation, atherogenesis, and blood clotting processes $[8,22,23,30]$. However, current knowledge does not allow a full definition of the contribution of leptin to the pathogenesis of DM2, and the results available from the literature are often contradictory and confusing.

With the increasing importance of diabetes and obesity detection, it is useful to reevaluate the role played by leptin in this conditions. Our study aimed at investigating circulating leptin concentrations in a group of patients with DM2, and at assessing in detail whether leptin concentrations correlate with selected biochemical and clinical parameters, as well as markers of systemic inflammation, in patients with DM2 and in healthy volunteers.

\section{MATERIALS I METHODS}

In our work, we analysed samples obtained from 71 patients diagnosed with type 2 diabetes, and treated at the Department of Endocrinology, Medical University of Lublin, Poland. The studied group (DM2) was comprised of 38 women $(53.5 \%)$ and 30 men (46.5\%), their mean age being $61.4 \pm 11.7$ years. What is more, the mean time of diabetes duration from diagnosis was $11.2 \pm 8.3$ years. According to World Health Organization (WHO) terminology and BMI classification [35], in our study-patient population, $\mathrm{n}=5$ patients were classified as having normal body weight $(7.0 \%), n=18$ patients $(25.4 \%)$ were overweight and $n=48$ patients $(67.6 \%)$ were obese. Additionally $n=65$ patients $(91.6 \%)$ were diagnosed with abdominal obesity based on IDF 2005 diagnostic criteria for this metabolic syndrome [37]. Patients with kidney failure, liver, heart and/or cardiovascular disease, cancer and acute and chronic inflammatory and autoimmune diseases were excluded for the study. The healthy control group (HC) consisted of 51 healthy subjects (30 women and 21 men) with a mean age of $57.8 \pm 13.7$ years, who were undergoing routine occupational health checks at the Department of Laboratory Diagnostics Teaching Hospital No 1, in Lublin, Poland.

All patients underwent clinical assessment, including the collection of medical history via direct interview and physical examination, and involving the collection of anthropometric measurements [height, weight, body mass index (BMI), and waist circumference], as well as measuring blood pressure.

Beyond the aforementioned, peripheral blood samples were collected at a single time point from each subject, in standard conditions (fasting state, collection between 8-10am). In every serum sample, basic laboratory measurements were performed [glucose, insulin, total cholesterol, HDL-cholesterol, triglycerides, creatinine, C-reactive protein (CRP)], as well as level of HbA1c in the whole blood. All analyses were performed using biochemical analyzer Roche Cobas 6000, and standard laboratory methods.

The levels of LDL-cholesterol were calculated using Friedewald formula [11] and glomerular filtration rate eGFR, using the simplified MDRD equation [15]. Insulin resistance status was evaluated from fasting glucose and insulin values, using the homeostasis model assessment (HOMA-IR) equation [19].

Leptin and IL-6 were determined via commercial enzyme-linked immunosorbent assays (ELISA); Human Leptin ELISA Clinical Range (BioVendor, Modrice, Czech Republic) and Human IL-6 ELISA Kit (Diaclone, Besançon, France), according to the manufacturer's instructions. The detection limit for Leptin ELISA kit was $0.2 \mathrm{ng} / \mathrm{ml}$, and intra-assay and inter-assay precision (CV) were $4.2-7.6 \%$, and $4.4-6.7 \%$, respectively. For the IL-6 ELISA kit, the detection limit was $2 \mathrm{pg} / \mathrm{ml}$, and the test was sensitive to native, as well as recombinant IL-6. Furthermore, there was no cross-reactivity with other human origin cytokines, such as: IL- $1 \alpha$, IL-1 $\beta$, IL-10, IL-12, IFN- $\gamma$, IL-4, TNF- $\alpha$, IL-8 and IL-13. The intra-assay and inter-assay precision (CV) were $4.2 \%$ and $7.7 \%$, respectively.

The study was ethically approved by Bioethical Committee at Medical University of Lublin (Poland). Informed consent was obtained from all subjects.

The statistical analysis of the results was performed using Statistica 10.0 StatSoft, and a p value that was less than or equal to 0.05 was considered statistically significant. The results were presented as a descriptive statistic - for quantitative variables: mean \pm standard deviation or median and interquartile range (IQR); - for qualitative variables: the number (n) and percentage (\%) of the reported cases. Distribution of measured variables was assessed using the Shapiro-Wilk's test. In addition, the Mann-Whitney U-test was conducted to compare the differences between groups. To assess which of the selected factors determined the differences in their levels of leptin between the two groups, we employed analysis of covariance ANCOVA. Furthermore, the simple relationship between leptin and selected variables were tested using the nonparametric Spearman rank correlation test. Finally, the complex relationship between leptin and the studied independent variables was analysed using multivariate linear regression. 


\section{RESULTS}

The concentration of leptin in DM2 patients was significantly higher than in $\mathrm{HC}(\mathrm{p}<0.01)$, with median value of 16.59 (IQR $8.58-33.39$ ) $\mathrm{ng} / \mathrm{ml}$ in DM2 vs median value of 6.66 (IQR 4.52-21.40) $\mathrm{ng} / \mathrm{ml}$ in HC. In the analysis of variance, higher leptin concentrations in DM2 group as compared to $\mathrm{HC}$, remained significant after adjusting for gender and age $(p<0.001)$, and were independent of HOMA-IR ( $p=0.003)$. However, differences in leptin levels between the groups disappeared when additional adjustments for anthropometric parameters (BMI, waist circumference) were applied $(\mathrm{p}=0.088)$.

The results of relationships analysis of leptin level with demographic, anthropometric and clinical parameters in both the DM2 group and controls are shown in Table 1.

Table 1. Correlation analysis of leptin concentration with clinical parameters, in a DM2 group and in a HC group

\begin{tabular}{|c|c|c|c|}
\hline \multicolumn{2}{|c|}{ Parameter } & DM2 $(n=71)$ & $\mathrm{HC}(n=51)$ \\
\hline \multicolumn{2}{|c|}{ Age (years) } & -0.107 & -0.029 \\
\hline \multirow{2}{*}{ Gender } & women & $\begin{array}{c}28.38 \neq \\
(20.39-38.72)\end{array}$ & $\begin{array}{c}13.14 \neq \\
(11.44-25.36)\end{array}$ \\
\hline & $\operatorname{men}^{R}$ & $\begin{array}{c}8.41 \\
(6.72-12.02) \\
\end{array}$ & $\begin{array}{c}4.53 \\
(2.63-6.49) \\
\end{array}$ \\
\hline \multicolumn{2}{|c|}{ Height $(\mathrm{m})$} & $-0.589 \neq$ & $-0.530+$ \\
\hline \multicolumn{2}{|c|}{ Body mass (kg) } & 0.166 & 0.125 \\
\hline \multicolumn{2}{|c|}{ BMI $\left(\mathrm{kg} / \mathrm{m}^{2}\right)$} & $0.544 \neq$ & $0.463+$ \\
\hline \multicolumn{2}{|c|}{ Waist circumference $(\mathrm{cm})$} & $0.274 *$ & 0.216 \\
\hline \multicolumn{2}{|c|}{ Systolic blood pressure $(\mathrm{mmHg})$} & 0.036 & -0.188 \\
\hline \multicolumn{2}{|c|}{ Diastolic blood pressure $(\mathrm{mmHg})$} & 0.041 & -0.002 \\
\hline \multicolumn{2}{|c|}{ Disease duration (years) } & $-0.273 *$ & na \\
\hline
\end{tabular}

Values are presented as: Spearman'a Rank correlation coefficient (R) for quantitative variables, median (intequarter range) for qualitative variables (statistical significance was analysed using the $U$ Mann-Whitney test);

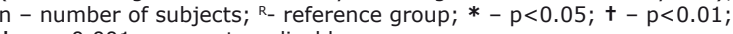
$\neq-\mathrm{p}<0.001$, na - not applicable

To compare leptin concentration in our patients with DM2 and controls, we examined men and women separately. We found significantly higher circulating leptin concentration $(\mathrm{p}<0.001)$ in women than in men with DM2, the median being 28.38 (IQR 20.39-38.72) $\mathrm{ng} / \mathrm{ml}$ vs median 8.41 (IQR $6.72-12.02) \mathrm{ng} / \mathrm{ml}$ for women and men, respectively. In the DM2 group, circulating leptin concentration correlated directly with BMI $(\mathrm{r}=0.544 ; \mathrm{p}<0.001)$ and waist circumference $(r=0.274 ; p<0.05)$. Additionally, this concentration was inversely correlated with height $(\mathrm{r}=-0.589 ; \mathrm{p}<0.001)$ and disease duration $(\mathrm{r}=-0.273 ; \mathrm{p}<0.05)$.

Similarly, in the HC group, a significantly higher leptin level was detected in women than in men, the median being 13.14 (IQR 11.44-25.36) ng/ml vs median 4.53 (IQR 2.63$6.49) \mathrm{ng} / \mathrm{ml}$, respectively. Leptin concentration also correlated inversely with height $(\mathrm{r}=-0.530 ; \mathrm{p}<0.01)$ and positively with BMI $(r=0.463 ; p<0.01)$ in this group.

The results of association analysis between leptin level and the biochemical parameters and inflammatory cytokines in the studied population are presented in Table 2.

Significant positive correlations were found in the DM 2 group between leptin level and CRP $(r=0.256 ; p<0.05)$ and IL- $6(\mathrm{r}=0.345 ; \mathrm{p}<0.01)$. In the $\mathrm{HC}$ group, positive relationships of leptin were detected with insulin concentration $(\mathrm{r}=0.464 ; \mathrm{p}<0.01)$, HOMA-IR $(\mathrm{r}=0.463 ; \mathrm{p}<0.01)$ and CRP level $(\mathrm{r}=0.449 ; \mathrm{p}<0.01)$. Moreover, leptin concentration in the $\mathrm{HC}$ group showed an inverse relationship with eGFR $(\mathrm{r}=-0.346 ; \mathrm{p}<0.05)$.

In the next stage of statistical analysis, multivariate linear regression was performed. Thirteen variables were selected and included in the analysis in order to find the independent factors affecting the variability of leptin. Forward stepwise regression was also performed, and the results are shown in Table 3. Among the selected variables, only gender and BMI were included in the predictive model explaining variability of leptin, and, in total, were responsible for $72.6 \%$ of the original variation of the studied adipocytokine.

Table 2. Correlation analysis of leptin and biochemical parameters and inflammatory cytokines, in a DM2 group and in a HC group

\begin{tabular}{|l|c|c|}
\hline \multicolumn{1}{|c|}{ Parameter } & DM2 $(\mathrm{n}=71)$ & $\mathrm{HC}(\mathrm{n}=51)$ \\
\hline Glucose $(\mathrm{mg} / \mathrm{dl})$ & -0.179 & 0.186 \\
\hline Insulin $(\mu \mathrm{U} / \mathrm{ml})$ & -0.068 & $0.464+$ \\
\hline HOMA-IR & -0.117 & $0.463+$ \\
\hline HbA1c $(\%)$ & -0.023 & -0.012 \\
\hline Total Cholesterol $(\mathrm{mg} / \mathrm{dl})$ & 0.014 & -0.092 \\
\hline HDL-Cholesterol $(\mathrm{mg} / \mathrm{dl})$ & 0.159 & 0.046 \\
\hline LDL-Cholesterol $(\mathrm{mg} / \mathrm{dl})$ & 0.025 & -0.099 \\
\hline Triglycerides $(\mathrm{mg} / \mathrm{dl})$ & -0.037 & 0.165 \\
\hline Creatinine $(\mathrm{mg} / \mathrm{dl})$ & -0.251 & -0.221 \\
\hline eGFR $\left(\mathrm{ml} / \mathrm{min} / 1.73 \mathrm{~m}^{2}\right)$ & -0.177 & $-0.346 *$ \\
\hline CRP $(\mathrm{mg} / \mathrm{l})$ & $0.256 *$ & $0.449+$ \\
\hline IL-6 $(\mathrm{pg} / \mathrm{ml})$ & $0.345+$ & 0.102 \\
\hline Results & &
\end{tabular}

Results are shown as: Spearman'a Rank correlation coefficient (R);

$\mathrm{n}$ - number of subjects;

$*-p<0.05 ;+-p<0.01$

Table 3. Results of multiple linear regression analysis for leptin as a dependent variable

\begin{tabular}{|c|c|c|c|c|c|c|}
\hline \multicolumn{7}{|c|}{$\begin{array}{c}\text { Dependent variable: Leptin }(\mathrm{ng} / \mathrm{ml}) \\
\mathrm{R}=0.858 ; \text { coefficient of determination } \mathrm{R} 2=0.735 ; \text { Corr. } \mathrm{R} 2=0.726 \\
\mathrm{~F}=75.025 ; \mathrm{SE} \text { of the estimate }=8.039 ; \mathrm{p}<0.0001 ; \mathrm{n}=122\end{array}$} \\
\hline $\begin{array}{l}\text { Independent } \\
\text { predictors }\end{array}$ & BETA* & SE. & BETA & SE. & $\mathrm{t}$ & $p$ \\
\hline Intercept & & & -35.971 & 6.145 & -5.854 & $<0.001$ \\
\hline Gender (women) & 0.560 & 0.071 & 17.110 & 2.173 & 7.874 & $<0.001$ \\
\hline BMI $\left(\mathrm{kg} / \mathrm{m}^{2}\right)$ & 0.556 & 0.071 & 1.456 & 0.186 & 7.820 & $<0.001$ \\
\hline
\end{tabular}

Independent predictors excluded from the model: age, waist

circumference, presence of diabetes type 2, concentrations of insulin, HDL - cholesterol, triglycerides, creatinine, CRP, IL-6, HOMA-IR, eGFR

\section{DISCUSSION}

The discovery of leptin in 1994 and the subsequent appreciation of its role in the regulation of appetite, body weight and energy balance has triggered major scientific interest in the potential contribution of this cytokine to the pathogenesis of DM2. However, the physiological mechanisms behind leptin synthesis, secretion, receptor binding and regulation of appetite, as well as energy expenditure are still not fully understood, yet involve numerous complex mechanisms including hormones, neurotransmitters and other regulatory pathways [7,22].

In this study, as expected, we found significantly higher leptin level in patients with DM2, in comparison with healthy subjects, even after adjustment for age and sex, and HOMA-IR. However, after adjusting for BMI and waist circumference, the difference in adipocytokine concentration between the two groups disappeared, suggesting the significant influence of obesity on the leptin level. Similar results were obtained by other researchers $[14,16,28]$. Due 
to the fact that this protein is involved in the modulation of many systems, and its secretion is subject to a complex and not fully-known mechanisms, it can be assumed that the association found in this study has a complex background.

The detailed multivariate linear regression analysis conducted in this study led to the conclusion that female gender and increased BMI were the main predictors of increased concentration of leptin in serum. Our results confirmed recently published findings $[16,21,27,28]$. The higher leptin concentration in women could be explained by greater subcutaneous fat content, as well as the influence of estrogens (which can stimulate leptin synthesis) [12]. Similarly to other studies on the role of leptin in metabolic disorders $[16,27,28,29]$, we showed the existence of a significant positive correlation between leptin levels and BMI and waist circumference in DM2 patients. The obtained results, and the fact that adipocytes are known to be the main sources of leptin synthesis, justify the assumption that leptin secretion increases when adipose tissue volume rises. Moreover, a number of experiments conducted to date have shown that body weight reduction may contribute to a significant reduction in systemic leptin levels [1,18], which, furthermore, suggests that changes in leptin concentration are rather the consequence of obesity and excess of adipose tissue than its cause. However, this is not in accordance with the known function of leptin as a hormone sensitive to excessive energy stores, which, in turn, activates the body metabolism so as to reduce body weight.

Several theories have been proposed to elucidate why in spite of elevated concentrations of leptin with anorexigenic properties, excess weight gain can be observed. The most known is the phenomenon of leptin resistance - where receptors have reduced reactivity to the leptin $[25,36]$. In our work, we showed higher leptin levels in patients with obesity associated DM2, and this situation remained in positive association with the amount of body fat. This result allows us to suggest the occurrence of the increased leptin resistance in our patients. It also confirms the notion that the leptin resistance observed in DM2 patients seems to be a phenomenon not only acting centrally (as in obesity), but also acting peripherally - indirectly contributing to the development of metabolic disturbances $[6,25,36]$.

Many authors have documented the role of elevated concentrations of leptin in the pathogenesis of insulin resistance and DM2 [9,26,27]: their results have shown the negative relationship of leptin with insulin, and HOMA-IR independently of age, gender and BMI in DM2 patients and in obesity. Indeed, Esteghamati et al. [10] concluded that the relationship of hyperleptynemia with DM2 is determined by insulin resistance and is independent of anthropometric parameters (BMI, waist circumference). Further analogous data were published by Wannamethee et al. [31] and Welsh et al. [33]. However, the results of our study show the opposite. According to Correia et al. [6], the impaired insulin sensitizing action of leptin, especially in relation to its role in the reduction of triglyceride accumulation in the skeletal muscle and pancreatic $\beta$ cells, may contribute to the development of insulin resistance and DM2. Hence, it is postulated, that the leptin resistance observed in obesity may become an additional component of the metabolic syndrome, and through the abolished insulin sensitizing action of leptin, be indirectly involved in its etiology. In our study, the positive correlation of leptin with the level of insulin and HOMA-IR was detected in the HC group, which also is in line with results reported by Lee et al. [14] and Silha et al. [26]. Similar results with HOMA-IR have been published by Zuo et al. [38] in a group of 1234 adults with normal glucose tolerance and BMI $23.5 \mathrm{~kg} / \mathrm{m} 2$. This further confirms that leptin can be a predictor of adverse metabolic changes and insulin resistance.

The results of research published to date strongly suggest that leptin plays a physiological role in the modulation of the inflammatory process, hence, highlighting its pro-inflammatory properties. Our results confirm that leptin is involved in inflammation, and this is in agreement with studies published by others $[5,14,28,32]$. In our work, we saw that the concentration of leptin increased with the burden of subclinical inflammation in patients with DM2, as evidenced by positive correlations of leptin with CRP and IL-6. The basis for this correlation is the immediate impact of the immune system on leptin production and secretion, and the fact that leptin induces pro-inflammatory cytokine synthesis (IL-6, IL-12, TNF-alpha) by monocytes and macrophages [22,23]. It, as well, increases the expression of CRP in human hepatocytes [5]. According to Chen et al. [5], the interaction between CRP and leptin may result in leptin resistance coming about by way of the direct inhibitory effect of CRP in binding leptin with its membrane receptors.

In our study, we also found a negative correlation of leptin level with eGFR in a HC group, which suggests that leptin is eliminated via kidneys. Thus, a decline in glomerular filtration rate may lead to impaired renal clearance of leptin and increase its concentration in plasma. This hypothesis was confirmed in the study by Saginova et al. [24], wherein obese patients in an early stage of chronic renal failure had significantly higher leptin levels, and a positive correlation of leptin existed with albuminuria, BMI, insulin, insulin resistance surrogate markers and CRP. This situation further indicates that the hyperleptynemia is not only related to the metabolic disorders, but also to impaired renal function. These observations were also evidenced by Wolf et al. [34], who demonstrated that leptin may have a direct impact on the pathophysiology of the kidneys. The experiments performed in this study showed that this adipocytokine stimulated the proliferation of glomerular mesangial cells, as well as increased the synthesis of transforming growth factor $\beta 1$ (TGF- $\beta 1$ ) and collagen production, resulting in progressive glomerulosclerosis and the presence of proteinuria. Moreover, currently available literature indicate that reasons for elevated levels of leptin in patients with chronic renal failure are not only the decrease in GFR, but also the coexisting inflammation and hyperinsulinemia [20]. Therefore, it is possible that hyperleptinemia may be involved in chronic inflammatory reactions leading to kidney damage.

Numerous studies to date have also revealed that elevated leptin levels may be an indicator of the risk of developing type 2 diabetes [4]. Our observations seemed to confirm these findings, as initial analysis of covariance showed that elevated levels of leptin are associated with DM2, regardless of age and sex, and HOMA-IR. However, after adjusting for 
additional anthropometric parameters, the above relationship disappears, which is consistent with reports by Ley et al. [16] and Sun et al. [28]. In contrast to our results, other studies previously cited here, by Esteghamati et al. [10] and Wannamethee et al. [31], demonstrated that leptin can predict the development of DM2 independently of BMI and waist circumference. It should also be noted that other controversial studies by Söderberg et al. [27] and Welsh et al. [33], which reported that leptin, a molecule being able directly influence $\beta$-cell activity, is an independent predictive factor of DM2 only in men. Recently published metaanalysis by Chen et al. [4] in 2014 also confirms the genderspecific differences in the relationship between circulating leptin levels and risk of DM2 in men (OR 1.37; 95\% CI $1.13-1.66 ; \mathrm{p}<0.05)$.

\section{CONCLUSION}

The results of this study have led to conclusion that leptin may participate in the complex pathogenesis of DM2 and be a predictor of the development of this disease. Since higher concentrations of leptin coexist with obesity, and correlate positively with markers of inflammation (CRP, IL-6), leptin levels should be considered in the pathogenesis of DM2. However, further studies, both lab-based and clinical are necessary to correctly understand the complex mechanisms linking leptin with a number of metabolic disturbances, in order to allow a clear definition of its role in the development of DM2.

\section{REFERENCES}

1. Anderlová K. et. al.: The influence of very-low-calorie-diet on serum leptin, soluble leptin receptor, adiponectin and resistin levels in obese women. Physiol. Res., 55(3), 277, 2006.

2. Bays H.E.: Adiposopathy is "sick fat" a cardiovascular disease? J. Am. Coll. Cardiol., 57(25), 2461, 2011.

3. Blüher M., Mantzoros C.S.: From leptin to other adipokines in health and disease: facts and expectations at the beginning of the 21st century. Metabolism, 64(1), 131, 2015.

4. Chen G.C., Qin L.Q., Ye J.K.: Leptin levels and risk of type 2 diabetes: gender-specific meta-analysis. Obes. Rev., 15(2), 134, 2014.

5. Chen K. et. al.: Induction of leptin resistance through direct interaction of C-reactive protein with leptin. Nat. Med., 12(4), 425, 2006.

6. Correia M.L., Rahmouni K.: Role of leptin in the cardiovascular and endocrine complications of metabolic syndrome. Diabetes Obes. Metab., 8(6), 603, 2006.

7. Dardeno T.A. et. al.: Leptin in human physiology and therapeutics. Front Neuroendocrinol., 31(3), 377, 2010.

8. Denroche H.C., Huynh F.K., Kieffer T.J.: The role of leptin in glucose homeostasis. J. Diabetes Investig., 3(2), 115, 2012.

9. Esteghamati A. et. al.: Association of serum leptin levels with homeostasis model assessment-estimated insulin resistance and metabolic syndrome: the key role of central obesity. Metab. Syndr. Relat. Disord. 7(5), 447, 2009.

10. Esteghamati A. et. al.: Serum visfatin is associated with type 2 diabetes mellitus independent of insulin resistant and obesity. Diabetes Res. Clin. Pract., 91(2), 154, 2011.

11. Friedewald W.T., Levy R.I., Fredrickson D.S.: Estimation of the concentration of low-density lipoprotein cholesterol in plasma, without use of the preparative ultracentrifuge. Clin. Chem., 18(6), 499, 1972.

12. Gui Y., Silha J.V., Murphy L.J.: Sexual dimorphism and regulation of resistin, adiponectin, and leptin expression in the mouse. Obes. Res., 12(9), 1481, 2004.
13. Kershaw E.E., Flier J.S.: Adipose tissue as an endocrine organ. J. Clin. Endocrinol. Metab,. 89(6), 2548, 2004.

14. Lee J.M. et. al.: The relationship between adipokines, metabolic parameters and insulin resistance in patients with metabolic syndrome and type 2 diabetes. Int. Med. Res., 37(6), 1803, 2009.

15. Levey A.S. et. al.: Using standardized serum creatinine values in the modification of diet in the renal disease study equation for estimating glomerular filtration rate. Ann. Intern. Med., 145(4), 247, 2006.

16. Ley S.H. et. al.: Adipokines and incident type 2 diabetes in an Aboriginal Canadian population: the Sandy Lake Health and Diabetes Project. Diabetes Care, 31(7), 1410, 2008.

17. Malecha-Jedraszek A. et. al.: Concentration of adiponectin in relation to insulin resistance in patients with type 2 diabetes. Ann. UMCS Sect. DDD, 24 (4), 73, 2011.

18. Martos-Moreno G.Á. et. al.: Effect of weight loss on high-molecular weight adiponectin in obese children. Obesity (Silver Spring),18(12), 2288, 2010 .

19. Matthews D.R. et. al.: Homeostasis model assessment: insulin resistance and beta-cell function from fasting plasma glucose and insulin concentrations in man. Diabetologia, 28(7), 412, 1985.

20. Menon V. et. al.: Factors associated with serum leptin in patients with chronic kidney disease. Clin. Nephrol., 61(3), 163, 2004.

21. Olstad R. et. al.: Leptin in the General Population, Differences in Sex Hormones, Blood Lipids, Gender and Life Style Characteristics. The Open Behavioral Science Journal, 5, 8, 2011.

22. Park H.K., Ahima R.S.: Physiology of leptin: energy homeostasis, neuroendocrine function and metabolism. Metabolism, 64(1), 24, 2015.

23. Paz-Filho G. et. al.: Leptin: molecular mechanisms, systemic proinflammatory effects, and clinical implications. Arq. Bras. Endocrinol. Metabol., 56(9), 597, 2012.

24. Saginova E.A. et. al.: The role of leptin, adiponectin and insulinresistance markers in development of early stages of chronic kidney disease and atherosclerosis of carotid arteries in obese patients. Ter. Arkh., 83(6), 47, 2011.

25. Sáinz N. et. al.: Leptin resistance and diet-induced obesity: central and peripheral actions of leptin. Metabolism, 64(1), 35, 2015.

26. Silha J.V. et. al.: Plasma resistin, adiponectin and leptin levels in lean and obese subjects: correlations with insulin resistance. Eur. J. Endocrinol., 149(4), 331, 2003.

27. Söderberg S. et. al.: Leptin predicts the development of diabetes in Mauritian men, but not women: a population-based study. Int. J. Obes. (Lond)., 31(7), 1126, 2007.

28. Sun Q. et. al.: Leptin and soluble leptin receptor levels in plasma and risk of type 2 diabetes in U.S. women: a prospective study. Diabetes, 2010; 611, 59(3).

29. Tokuyama Y. et. al.: Serum resistin level is associated with insulin sensitivity in Japanese patients with type 2 diabetes mellitus. Metabolism, 56(5), 693, 2007.

30. Wang H., Luo W., Eitzman D.T.: Leptin in thrombosis and atherosclerosis. Curr. Pharm. Des., 20(4), 641, 2014.

31. Wannamethee S.G. et. al.: Adipokines and risk of type 2 diabetes in older men. Diabetes Care, 30(5), 1200, 2007.

32. Wannamethee S.G. et. al.: Plasma leptin: associations with metabolic, inflammatory and haemostatic risk factors for cardiovascular disease. Atherosclerosis, 191(2), 18, 2007.

33. Welsh P. et. al.: Leptin predicts diabetes but not cardiovascular disease: results from a large prospective study in an elderly population. Diabetes Care, 32(2), 308, 2009.

34. Wolf G., Ziyadeh F.N.: Leptin and renal fibrosis. Contrib. Nephrol., 151, 175, 2006.

35. World Health Organisation: Obesity: preventing and managing the global epidemic. Report of a WHO consultation, Genewa 1997.

36. Zhou Y., Rui L.: Leptin signaling and leptin resistance. Front Med., 7(2), 207, 2013.

37. Zimmet P., Alberti K.G., Serrano Ríos M.: A new international diabetes federation worldwide definition of the metabolic syndrome: the rationale and the results. Rev. Esp. Cardiol., 58(12), 1371, 2005.

38. Zuo H. et. al.: Association between serum leptin concentrations and insulin resistance: a population-based study from China. PLoS One, 8(1), 1, 2013. 